\title{
Role of screening in the density functional applied to transition-metal defects in semiconductors
}

\author{
Viktor Ivády, Igor Abrikosov, Erik Janzén and A Gali
}

\section{Linköping University Post Print}

\section{Tweet}

N.B.: When citing this work, cite the original article.

Original Publication:

Viktor Ivády, Igor Abrikosov, Erik Janzén and A Gali, Role of screening in the density functional applied to transition-metal defects in semiconductors, 2013, Physical Review B. Condensed Matter and Materials Physics, (87), 20.

http://dx.doi.org/10.1103/PhysRevB.87.205201

Copyright: American Physical Society http://www.aps.org/ 


\title{
Role of screening in the density functional applied to transition-metal defects in semiconductors
}

\author{
Viktor Ivády, ${ }^{1,2, *}$ I. A. Abrikosov, ${ }^{1}$ E. Janzén, ${ }^{1}$ and A. Gali ${ }^{2,3, \dagger}$ \\ ${ }^{1}$ Department of Physics, Chemistry and Biology, Linköping University, SE-581 83 Linköping, Sweden \\ ${ }^{2}$ Wigner Research Centre for Physics, Hungarian Academy of Sciences, PO Box 49, H-1525 Budapest, Hungary \\ ${ }^{3}$ Department of Atomic Physics, Budapest University of Technology and Economics, Budafoki út 8., H-1111 Budapest, Hungary
}

(Received 15 August 2012; revised manuscript received 27 March 2013; published 1 May 2013)

\begin{abstract}
We study selected transition-metal-related point defects in silicon and silicon carbide semiconductors by a range-separated hybrid density functional (HSE06). We find that HSE06 does not fulfill the generalized Koopmans' theorem for every defect, which is due to the self-interaction error in the functional in such cases. Restoring the so-called generalized Koopmans' condition with a simple correction in the functional can eliminate this error and brings the calculated charge transition levels remarkably close to the experimental data as well as to the calculated quasiparticle levels from many-body perturbation theory.
\end{abstract}

DOI: 10.1103/PhysRevB.87.205201

PACS number(s): 71.15.Mb, 61.72.J-, 61.82.Fk, 76.30.-v

\section{INTRODUCTION}

Tractable solutions for the problem of many-electron systems are highly needed in materials science and are under intense research. One key example of such problems is the treatment of small imperfections in single crystals, i.e., point defects in semiconductors. Point defects can completely change locally the chemical bonds of the host crystal, thus $a b$ initio methods are needed in order to determine their geometry, electronic structure, ionization energies, or optical excitations. The most challenging type of point defects is the transition-metal-related (TM-related) defects in traditional semiconductors where $d$ orbitals tightly localized on the TM atoms and $s p^{3}$ hybrid orbitals are both present in the system. It is known from, e.g., studies of Mott insulators that $d$-electron systems may be highly correlated whereas the electronic structure of traditional semiconductors can phenomenologically be well described by independent-particle theories. Simulations of TM defects in semiconductors are therefore a complex problem; thus, it is an obvious system with which to investigate the predictive power of the ab initio methods.

Density functional theory (DFT) is the most widespread technique for first-principles calculation in condensed matter physics. Particularly, DFT has been proven to be an extremely powerful tool to study defects in semiconductors. ${ }^{1}$ The success of DFT calculations is based on well-developed approximate (semi)local functionals ${ }^{2-5}$ that make it possible to study relatively large systems at moderate computational cost with a surprisingly good accuracy. ${ }^{1}$ The success of the commonly used (semi)local functionals might be unexpected because they suffer from the self-interaction error $^{3}$ and from the absence of the derivative discontinuity of the exchange-correlation potential, which results in the underestimation of the band gap of semiconductors. The predictive power of (semi)local functionals is thus restricted for ionization energies of defects in semiconductors and can even fail to correctly describe the nature of the host semiconductor or the defect state in the semiconductor in pathological cases. ${ }^{6-8}$ By mixing a nonlocal Fock exchange into the density functional, i.e., by using hybrid density functionals, one can restore the band gap of host semiconductors via tuning the mixing parameter. $^{9-13}$ Alternatively, by introducing a range-separated hybrid density functional (HSE06), it is found that, with a fixed mixing parameter and range of separation, the band gap of many semiconductors with $s p^{3}$ hybrid orbitals can be well reproduced ${ }^{14}$ and, in addition, the structural parameters, redox reaction energies, and formation energies of transition-metal compounds can also be well accounted for by HSE06. ${ }^{15}$ This brings a hope that HSE06 is able to predict the ground state $^{16-18}$ and ionization energies for TM-related defects in semiconductors that contain both $d$ and $s p^{3}$ orbitals. However, the consistency of the approach requires the use of the same mixing and range-separation parameters for the host and impurity states. It has been observed that this requirement makes it difficult to describe band structure and defect states simultaneously. ${ }^{19,20}$

In this paper we study TM defects in silicon ( $\mathrm{Si}$ ) and $4 H$ silicon carbide ( $\mathrm{SiC}$ ) semiconductors by the HSE06 functional using plane-wave large-supercell calculations, for those experimental data about their structure and ionization energies are available. We show that HSE06 can qualitatively fail in such complex systems due to insufficient screening of the Coulomb interaction between localized $d$ electrons, leading to incomplete cancellation of the self-interaction error which manifests itself as disobeying the generalized Koopmans' theorem $(\mathrm{gKT}){ }^{7,19,21,22}$ We suggest a correction scheme for the hybrid functional which fulfills the conditions of the gKT. The corrected functional allows for a simultaneous description of localized and extended states, is first principles in nature, and brings our theoretical results remarkably close to the experimental data as well as to the results obtained by means of many-body perturbation theory. Our work underlines the importance of testing a fulfillment of gKT for the applied functionals on a given many-electron system.

This paper is organized as follows: Section II A describes the details of the calculations. In Sec. II A we introduce the non-Koopmans' energy used to indicate the errors, which appear in hybrid functional calculations due to the selfinteraction error, and describe our correction scheme, the $\mathrm{HSE06}+\mathrm{V}_{\mathrm{w}}$ method. Section III presents results and a discussion of our calculations using the $G W$ approximation, the DFT HSE06 hybrid functional, and our corrected functional $\mathrm{HSE06}+\mathrm{V}_{\mathrm{w}}$. In Sec. IV we summarize our findings and results. 


\section{METHODOLOGY}

\section{A. Calculations}

We select interstitial $\mathrm{Fe}$ in $\mathrm{Si}\left(\mathrm{Fe}_{\mathrm{i}}\right)$ and titanium $\left(\mathrm{Ti}_{\mathrm{Si}}\right)$, vanadium $\left(\mathrm{V}_{\mathrm{Si}}\right)$, and chromium $\left(\mathrm{Cr}_{\mathrm{Si}}\right)$ substituting in a Si site in $4 \mathrm{H} \mathrm{SiC}$. For all of these defects, the structure and the corresponding ionization energies are well established from experiments. $^{23-26}$ In addition, we investigate tungsten (W) in $4 H \mathrm{SiC}$ because $\mathrm{W}$-related ionization energies have been detected recently. ${ }^{27}$ In this case, the origin of the W-related centers are not ambiguously identified. According to our previous study, ${ }^{28}$ both the $\mathrm{Si}$-substitution site $\left(\mathrm{W}_{\mathrm{Si}}\right)$ and the asymmetric split vacancy configuration $\left(\mathrm{W}_{\mathrm{ASV}}\right)$ may be stable, thus both defects are considered. For the electronic structure calculations we use the VASP package ${ }^{29}$ with the plane-wave basis (cutoff of $420 \mathrm{eV}$ ) and the projector augmented wave $(\mathrm{PAW})^{30}$ method for the ion cores. In the case of metal atoms we utilize small-core PAW projectors. In order to model the $4 H \mathrm{SiC}$ and $\mathrm{Si}$ host we use 576-atom and 512-atom supercells, respectively. In the large supercells the Brillouin zone is sampled at $\Gamma$ point. For comparison with the experimental result the spurious size dependence of the total energies of our supercell calculations is eliminated by charge correction ${ }^{31}$ with the order of magnitude $0.1 \mathrm{eV}$. The geometry of the defects is optimized by the HSE06 functional until the forces acting on the atoms are less than $0.01 \mathrm{eV} / \AA$.

In order to examine the performance of the HSE06screened nonlocal hybrid density functional on the selected transition-metal defects in semiconductors we calculate the adiabatic ionization energies or charge transition levels of these defects:

$$
\varepsilon\left(q \mid q^{\prime}\right)=\left[E_{q^{\prime}}+E^{\mathrm{corr}}\left(q^{\prime}\right)\right]-\left[E_{q}+E^{\mathrm{corr}}(q)\right]-\varepsilon_{\mathrm{CBM}},
$$

where $E_{q}$ is the total energy, $E^{\text {corr }}(q)$ is the charge correction of charge state $q$, and $\varepsilon_{\mathrm{CBM}}$ is the conduction-band minimum (see Ref. 1 for more details). The HSE06 functional gives the proper description of the band structure of the semiconductor host. The band-gap error is within $0.1 \mathrm{eV}$ for the pristine $4 H$ $\mathrm{SiC}$ and $\mathrm{Si}$ host which causes an uncertainty in the calculated $\varepsilon\left(q \mid q^{\prime}\right)$ of about $0.1 \mathrm{eV}$.

In order to even further examine the accuracy of the approximations included in HSE06 functional, we carry out sophisticated parameter-free $G W_{0}$ calculations ${ }^{32}$ on defective supercells with 128 atoms and HSE06 relaxed geometry. We apply 1344 bands in the calculation of the response function, and the Brillouin zone is sampled with $2 \times 2 \times 2$ Monkhorst-Pack $k$-point set. ${ }^{33}$ The starting wave functions are obtained from the HSE06 calculation, then the Green-function $G$ and the wave functions are self-consistently updated while keeping the screened Coulomb interaction $W$ fixed. We find that four iterations are sufficient to reach the self-consistent quasiparticle levels within $0.05 \mathrm{eV}$.

\section{B. Non-Koopmans' energy and the correction potential}

It is well known that self-Hartree and the exchange potentials do not cancel each other in (semi)local DFT functionals. In (semi)local functionals the resultant self-repulsive potential lowers the localization degree of the states and causes the spurious occupation dependence of the Kohn-Sham energies as well as the spurious, generally convex, curvature of the total energy with respect to fractional occupations. ${ }^{7,21,22}$ The opposite behavior is characteristic for the Hartree-Fock (HF) method. By mixing HF and semilocal exchange, the self-interaction error is reduced in hybrid functionals. Unfortunately, it is not eliminated completely.

Previous studies show ${ }^{7,19,21,22}$ that one of the quantitative manifestations of the self-interaction error in DFT functionals is the discrepancy between the Kohn-Sham eigenvalue of the highest-occupied state and the corresponding ionization energy. This energy difference is usually called the nonKoopmans' energy,

$$
E_{\mathrm{NK}}=\varepsilon_{N}-E_{I}=\varepsilon_{N}-\left(E_{N}-E_{N-1}\right),
$$

where $\varepsilon_{N}$ is the Kohn-Sham quasiparticle energy of a localized state in an $N$-electron system, $E_{I}$ is the ionization energy of the system which equals to the difference of the total energies $E_{N}$ and $E_{N-1}$ of the $N$-electron and the $(N-1)$-electron systems, respectively. In HF theory the Koopmans' theorem states that the single-particle energies equal the ionization energy for every occupied state, while in DFT this statement is only valid for the highest-occupied state with an exact exchange-correlation functional. In the case of an exact functional, the condition $E_{\mathrm{NK}}=0$ should be fulfilled, which is the so-called generalized Koopmans' condition (gKC). Due to Janak's and Slater's theorems, other important features of the exact functional is simultaneously remedied: linear behavior of the total energy and constant behavior of the (highest-occupied) single-particle level with respect to fractional occupation are also fulfilled. The last condition explicitly shows that the single-particle state does not suffer from any occupation-dependent potential and thus represents a self-interaction-free functional.

Here, we would like to emphasize that Eq. (2) examines the change in energies because of the change of the electronic structure induced by the addition of one electron to the system. Due to this, one has to omit those effects that can change the total energy in other ways. Such an effect is the geometry relaxation. Due to this we calculated Eq. (2) from total energies determined on the fixed geometry of the initial charged state.

As we show later, the states of the transition-metal impurities described by the HSE06 functional may possess self-interaction which hinder the accessibility of accurate theoretical results. To remedy this shortcoming we applied an occupation-dependent potential

$$
V_{w}^{I m}=\frac{w}{2}\left(1-2 n_{m}^{I}\right)
$$

on the $d$ orbitals together with the screened nonlocal HSE06 functional. In Eq. (3), $w$ is a parameter for the strength of the potential, whose physical meaning will become clear below and which is determined self-consistently by satisfying $\mathrm{gKC}: E_{\mathrm{NK}}=0$. We emphasize that the resultant functional is ab initio in the sense that it is not fit to any empirical parameter. We note that the form of Eq. (3) is equivalent to Dudarev's implementation of the LDA $+U$ method, ${ }^{34}$ and therefore it is straightforward to use the suggested scheme with existing first-principles package like VASP.

The occupation number $n_{m}^{I}$ in the VASP implementation is determined as a projection of the wave function of the system 
on the spherical harmonics $l m$ on atom site $I$, where $l=2$ in our case.

\section{RESULTS AND DISCUSSION}

\section{A. Accuracy of HSE06}

From the comparison of the experimental data with the results of HSE06 calculations, shown in Fig. 1 and Table I, it is apparent that HSE06 cannot predict the correct charge transition levels for all the defects. The magnitude of the discrepancies depend on the TMs as well as the charge transition level (Table I). Particularly, the calculated ionization energies of $\mathrm{Cr}$ strikingly differs from the experimental values and are qualitatively wrong.

Let us demonstrate that the failure of HSE06 for some defects originates from the incorrect treatment of the atomiclike $d$ orbitals of TM atoms. In Table I one can see the difference in the calculated HSE06 results and experimental data as well as the calculated non-Koopmans' energy for the defect state that gets occupied through the charge transition. The results clearly show that this quantity correlates with the discrepancy between HSE06 results and experimental data for the charge transition levels. The obvious conclusion of this finding is that the errors originate from the spurious self-interaction which still remains in the HSE06 functional.

In the HSE06 functional, the mixing parameter and the range of separation play the role of effectively screening the Coulomb interaction, ${ }^{35-37}$ and their variation in practice interpolates between the substantial underestimation of the localization effects in (semi)local functionals and their overestimation in Hartree-Fock theory. But the strength of the screening should itself depend on the localization degree of

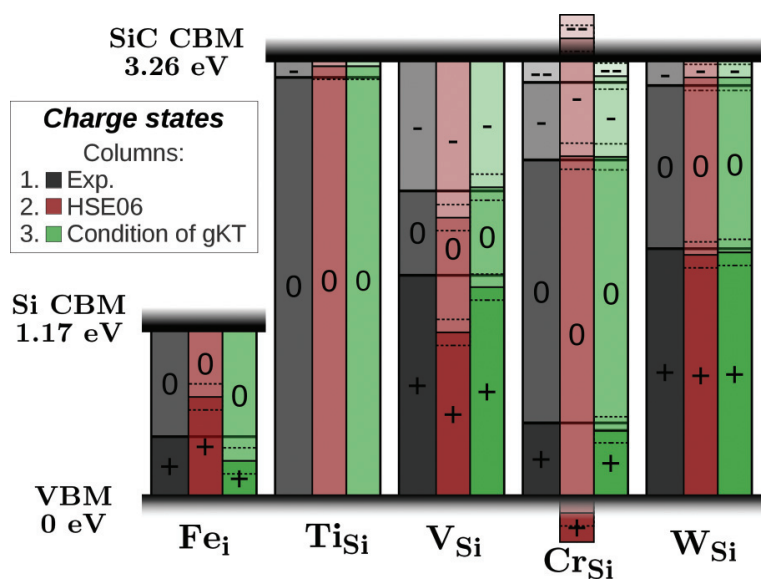

FIG. 1. (Color online) Measured and theoretically predicted charge transition levels of transition metal impurities in $4 H \mathrm{SiC}$ and $\mathrm{Si}$ hosts. In all cases the experimental results ${ }^{23-25,27}$ are represented with dark gray (first) columns where the thick horizontal line represents the charge transition levels. The results of HSE06 calculations and our correction method with gKT satisfied are represented with red (second) columns and light green (third) columns, respectively, where thin horizontal lines provide the calculated charge transition levels. The bars indicated by dashed lines around the calculated charge transition levels show the inherent uncertainty of the calculation. Chromium needs special consideration regarding the $(0 \mid-)$ transition level; see text.
TABLE I. Experimental values of charge transition levels $\left(\varepsilon_{\text {expt }}\right)$, deviations of the theoretically predicted values from experiment for transitional-metal-related point defects $\mathrm{Ti}_{\mathrm{Si}}, \mathrm{V}_{\mathrm{Si}}, \mathrm{Cr}_{\mathrm{Si}}$, and $\mathrm{W}_{\mathrm{Si}}$ in $4 \mathrm{H}-\mathrm{SiC}$ and $\mathrm{Fe}_{\mathrm{i}}$ in $\mathrm{Si}$. In the calculations, the HSE06 functional and a corrected HSE06 functional (see text) are used. The finite values of $E_{\mathrm{NK}}$ clearly indicate the presence of a self-interaction error in the HSE06 functional $\left(\Delta \varepsilon_{\mathrm{HSE} 06}\right)$. When a small value of $E_{\mathrm{NK}}$ is obtained $\left(E_{\mathrm{NK}}^{\prime}\right)$ with the help of an additional correction functional $\left(\mathrm{V}_{\mathrm{w}}\right)$ with a strength parameter $w$, the discrepancy $\left(\Delta \varepsilon_{\mathrm{HSE06}+\mathrm{V}_{\mathrm{w}}}\right)$ goes below $0.1 \mathrm{eV}$ which is the uncertainty in our calculation. Bold (italic) numbers represent the case when the calculated charge transition level is out of (within) the error bar. The data in all columns are in units of $\mathrm{eV}$.

\begin{tabular}{lcccccc}
\hline \hline Transition & $\varepsilon_{\text {expt }}$ & $E_{\mathrm{NK}}$ & $\Delta \varepsilon_{\mathrm{HSE} 06}$ & $w$ & $E_{\mathrm{NK}}^{\prime}$ & $\Delta \varepsilon_{\mathrm{HSE} 06+\mathrm{V}_{\mathrm{w}}}$ \\
\hline $\mathrm{Ti}_{\mathrm{Si}}:(0 \mid-)$ & $-0.12^{\mathrm{a}}$ & -0.02 & +0.01 & 0.0 & -0.02 & +0.10 \\
$\mathrm{~V}_{\mathrm{Si}}:(+\mid 0)$ & $-1.60^{\mathrm{a}}$ & $\mathbf{- 0 . 9 1}$ & $\mathbf{- 0 . 4 3}$ & -2.7 & +0.02 & -0.09 \\
$\mathrm{~V}_{\mathrm{Si}}:(0 \mid-)$ & $-0.97^{\mathrm{a}}$ & $-\mathbf{0 . 8 1}$ & $\mathbf{- 0 . 2 0}$ & -2.2 & +0.02 & +0.03 \\
$\mathrm{Cr}_{\mathrm{Si}}:(+\mid 0)$ & $-2.70^{\mathrm{a}}$ & & $\mathrm{VB}$ & -3.0 & -0.00 & -0.05 \\
$\mathrm{Cr}_{\mathrm{Si}}:(0 \mid-)$ & $-0.74^{\mathrm{a}}$ & -0.21 & +0.02 & & & +0.01 \\
$\mathrm{Cr}_{\mathrm{Si}}:(-\mid 2-)$ & $-0.18^{\mathrm{a}}$ & $-\mathbf{1 . 8 8}$ & $+\mathbf{1 . 8 5}$ & -6.0 & +0.01 & +0.05 \\
$\mathrm{~W}_{\mathrm{Si}}:(+\mid 0)$ & $-1.40^{\mathrm{b}}$ & -0.13 & -0.05 & -1.2 & +0.01 & -0.03 \\
$\mathrm{~W}_{\mathrm{Si}}:(0 \mid-)$ & $-0.18^{\mathrm{b}}$ & -0.03 & +0.06 & 0.0 & -0.03 & +0.06 \\
$\mathrm{Fe}_{\mathrm{i}}:(+\mid 0)$ & $-0.79^{\mathrm{c}}$ & $-\mathbf{0 . 6 8}$ & $\mathbf{+ 0 . 3 2}$ & -3.8 & $\mathbf{- 0 . 0 9}$ & $-\mathbf{0 . 1 5}$ \\
\hline \hline
\end{tabular}

${ }^{\mathrm{a}}$ Ref. 24.

${ }^{\mathrm{b}}$ Ref. 27.

${ }^{c}$ Ref. 23.

the states. In the case of TM impurities the strong repulsive interaction between the electrons stems the extension of the atomic-like electron states. In such a strongly correlated system, the localized states are more favorable than the extended state. Due to the large localization degree of the $d$ states with respect to that of host $s p^{3}$ hybrid orbitals, in general it should not be possible to describe both subsystems with the same values of parameters in a hybrid functional. Lany and Zunger ${ }^{19}$ demonstrated that one should not expect that adjusting parameters of hybrid functionals would automatically give accurate results for both host band gaps and defect levels and pointed out homogeneous screening of the Fock exchange in hybrid functionals as a serious simplification. Still, it is desirable to have an option to describe the orbitals with different degrees of localization in calculations.

At this stage, it is important to mention that a systematic study of the role of nonlocal exchange in the electronic structure of correlated oxides by Iori et al. ${ }^{37}$ demonstrates that the default values of the parameters of the HSE06 functional systematically overestimate band gaps in these materials, indicating insufficient screening of the Coulomb interaction. Negative values of $E_{\mathrm{NK}}$ in Table I also indicate that the screening of the Coulomb interaction is insufficient. ${ }^{19}$

In order to prove the self-interaction error of the HSE06 functional, we carry out $G W_{0}$ calculations $^{32}$ on defective supercells. The observed quasiparticle correction (see Table II) confirmed the over-localization error of the HSE06.

\section{B. Analysis of proposed correction scheme}

Considering that $E_{\mathrm{NK}}$ is an appropriate measure to construct a self-consistent correction method to eliminate the 
TABLE II. The position of the highest-occupied single-particle levels of transitional-metal defects in the gap with respect to the conduction band edge (CBM) in units of eV. The table shows the results of calculations with the $G W_{0}$ method, the HSE06 functional, and the corrected HSE06 functional (HSE06 $+\mathrm{V}_{\mathrm{w}}$ ). In the case of $\mathrm{Cr}_{\mathrm{Si}}(0)$ the corresponding single-particle state falls in the valence band shown as "VB," thus $G W_{0}$ started far from the true ground state of the system. When non-Koopmans' energy $\left(E_{\mathrm{NK}}\right)$ is not negligible the correction method as well as the $G W_{0}$ method shift the levels up by approximately the same value. This result confirms the presence of the self-interaction error in the HSE06 functional and validates our corrected functional.

\begin{tabular}{lccc}
\hline \hline Defect & $G W_{0}$ & HSE06 & HSE06 $+\mathrm{V}_{\mathrm{w}}$ \\
\hline $\mathrm{V}_{\mathrm{Si}}(0)$ & -1.86 & -2.79 & -1.94 \\
$\mathrm{~V}_{\mathrm{Si}}(-)$ & -1.21 & -2.19 & -1.50 \\
$\mathrm{~W}_{\mathrm{Si}}(0)$ & -1.66 & -1.85 & -1.68 \\
$\mathrm{~W}_{\mathrm{Si}}(-)$ & -0.94 & -0.83 & -0.83 \\
$\mathrm{Cr}_{\mathrm{Si}}(0)$ & -3.22 & $\mathrm{VB}$ & -2.83 \\
$\mathrm{Cr}_{\mathrm{Si}}(-)$ & -0.74 & -1.00 & \\
$\mathrm{Cr}_{\mathrm{Si}}(2-)$ & +0.07 & -0.49 & -0.17 \\
\hline \hline
\end{tabular}

self-interaction error in the functional, ${ }^{7,21}$ we propose a scheme that does not use any empirical parameters to counteract the error due to insufficient screening of the Coulomb interaction between $d$ electrons localized at TM-related defects, which is apparently present in the HSE06 functional.

In our correction technique, as described earlier, the occupation-dependent potential Eq. (3) is applied on the $d$ orbitals together with the screened nonlocal HSE06 functional, denoted as HSE06 $+\mathrm{V}_{\mathrm{w}}$.

The results are summarized in Table I. One sees that $\mathrm{gKC}$ could be fulfilled with great accuracy $\left(E_{\mathrm{NK}}^{\prime}\right)$ in all cases except for $\mathrm{Fe}_{\mathrm{i}}$ in $\mathrm{Si}$ and $\mathrm{Cr}_{\mathrm{Si}}:(0 \mid-)$ in $\mathrm{SiC}$. Note that achieving $\mathrm{gKC}$ with a certain accuracy causes the corrected single-particle level to be independent from the occupation number with the same accuracy, indicating the self-interaction-free description of the defect state. The charge transition level is obtained after the geometry relaxation of the system with the use of the corrected HSE06 $+\mathrm{V}_{\mathrm{w}}$ functional shown in the last column of Table I. Apparently, this correction method can bring the theoretical values closer to the experimental values in every case. When $\mathrm{gKC}$ is accurately satisfied the calculated levels approach the experimental levels within $0.1 \mathrm{eV}$, which is the expected uncertainty in our calculation. Also, it can be seen that when $\mathrm{gKC}$ is fulfilled the position of the defect state in the gap is close to the corresponding quasiparticle level calculated by the $G W_{0}$ approximation of the many-body perturbation theory (see Table II).

In the case of iron, $E_{\mathrm{NK}}$ has an extrema and does not reach zero. We observed that the particular defect state is not so well localized on the Fe $d$ orbital in the $\mathrm{Fe}_{\mathrm{i}}$ defect, thus our correction scheme is not sufficiently effective. A similar effect can be found in case of $\mathrm{Cr}_{\mathrm{Si}}$. In the ground state of the negatively charged $\mathrm{Cr}_{\mathrm{Si}}$ defect, a double-degenerate $e$ level and an $a_{1}$ level appear in the gap, originating from the split $d$ orbitals of $\mathrm{Cr}$ in the crystal field of $4 \mathrm{H}-\mathrm{SiC}$ with $\mathrm{C}_{3 \mathrm{v}}$ symmetry. In the neutral charge state two electrons occupy the $e$ state while the $a_{1}$ state is empty, forming an $S=1$ spin state. Due to the strong correlation of the $d$ orbitals, the $S=3 / 2$ state is formed in the negatively charged state where three electrons occupy the $e$ and $a_{1}$ levels with parallel spins. In the negatively charged state, our correction method acts on the highest occupied $a_{1}$ level which is less atomic like compared to the $e$ level. In Table I the $\mathrm{Cr}_{\mathrm{Si}}:(0 \mid-)$ charge transition level is calculated as $\varepsilon_{G W}+E^{\text {relax }}+E^{\text {corr }}$, where $\varepsilon_{G W}$ is the highest-occupied quasiparticle level in the negative charge state, and $E^{\text {relax }}$ is the relaxation energy of the defect due to ionization. In the case of the $\mathrm{Cr}_{\mathrm{Si}}:(-\mid 2-)$ transition, the $a_{1}$ level becomes empty and the $e$ level gets fully occupied by ionizing from () to (2) states. Here, we set $S=1 / 2$ instead of the energetically favorable $S=3 / 2$, for the initial negative charge state in order to be able to study of the effect of the occupation of the $e$ level and to correct it by satisfying gKC with our correction functional.

Considering the strength of the required correction potentials, an important remark can be made. In all the cases when the correction is needed, the parameter $w$ is negative, representing a repulsive potential for occupied states. This observation confirms that the HSE06 functional over-localizes the defect state of the TM impurities, while this spurious effect is eliminated with our technique. In more detail this is illustrated in Fig. 2(b) where the difference in charge density of the HSE06 and HSE06 $+\mathrm{V}_{\mathrm{w}}$ calculations is shown. In the case of TM impurities, the HSE06 functional clearly represents an overcorrection with respect to standard (semi)local DFT functionals. We also note that the actual value of $w$ in our correction scheme depends on the chosen ion potentials.

In order to understand the physical meaning of the parameter $w$ in Eq. (3), we will make use of the similarity between the way hybrid functionals and the LDA $+U$ approach $^{34,38,39}$ correct the self-interaction error, as pointed out by several groups. ${ }^{37}$ For the case of correlated electrons it becomes particularly apparent within the so-called exact exchange for the correlated-electron method by Novak and coworkers. ${ }^{40}$ The LDA $+U$ functional was introduced in order to take into account the effects of the strong correlation which is usually not properly described by (semi)local functionals. ${ }^{34,38,39}$

The positive value of the Hubbard parameter $U$ representing the strength of the screened Coulomb potential makes the atomic-like states more favorable and increases the localization (a)

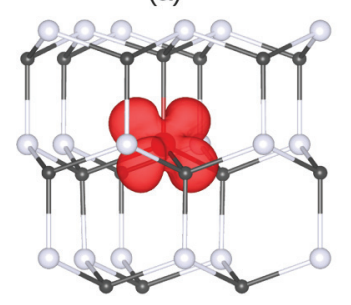

(b)

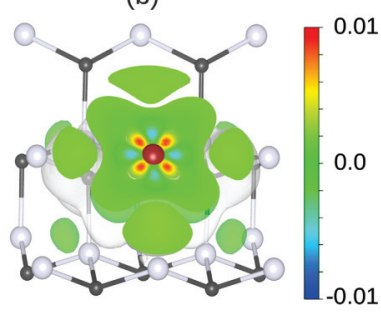

FIG. 2. (Color online) (a) Calculated electron density (with isosurface value of 0.05 ) of unpaired $d$ orbital of neutral vanadium substitutional defect in $4 \mathrm{H} \mathrm{SiC}$. (b) Two-dimensional plot of differences between two calculated charge densities using HSE06 functional and corrected HSE06 functional around the vanadium atom. The positive finite density clearly represents the overlocalization error of the HSE06 functional. 
of the states on atomic orbitals. In practice, the screening of $U$ is achieved by a reduction of the large bare Coulomb value, and the actual strength of the on-site electron repulsion can be either determined empirically ${ }^{41,42}$ or evaluated $a b$ initio. ${ }^{43,44}$ As a matter of fact, in our case of the TM defects, the Coulomb interaction between localized electrons (Fig. 2) appears to be insufficiently screened in the HSE06 functional due to its homogeneous nature and parameters that are adjusted to describe more extended states. In the LDA $+U$ formalism this error could be corrected by a reduction of the parameter $U$. Employing the above-mentioned similarity between the hybrid functionals and the LDA $+U$ method, we see that the action of the occupation-dependent potential in Eq. (3) on localized orbitals at impurity sites effectively increases the screening of the Coulomb interaction between electrons occupying these orbitals.

\section{Relative stability of defects}

As showed earlier, accurate charge transition levels can be obtained by our correction scheme from the sum of the corrected vertical ionization energy and the geometry relaxation energy due to ionization (see Fig. 1 and Table I). The necessary correction of the defect states depends on the given transitional metal as well as on the total occupation number of the $d$-like states of this atom or, in other words, on the charge state of the defect. Due to the varying additional screening functional, the total energy is not uniquely defined. The main difficulty may arise from this behavior when the total energies of different configurations of a given TM impurity are compared in order to determine their relative stability.

To overcome this difficulty, it is possible to choose a common screening parameter $w_{\mathrm{c}}$ for two or more different configurations of the impurity. A rational choice is the average of the parameters $w$ obtained for the different configurations. Note that, because the charge transition levels are well defined in our correction technique, it is sufficient to consider the problem of relative stability of one charge state; for example, the neutral state, of the defects.

We studied the formation energies of the experimentally most relevant TM defects in $4 H-\mathrm{SiC}$. We considered the substitutional defect $\left(\mathrm{TM}_{\mathrm{Si}}\right)$ at an $h$ site and the asymmetric split vacancy defect (ASV), built up from the pair $\mathrm{TM}_{\mathrm{Si}}$ and a carbon vacancy $\left(\mathrm{TM}_{\mathrm{Si}}-\mathrm{V}_{\mathrm{C}}\right)$ at an $h h$ site when $\mathrm{TM}$ is $\mathrm{V}$ or $\mathrm{W}$.

TABLE III. Optimized screening parameter $w$ that fulfills the $\mathrm{gKC}$ and the corresponding corrected charge transition levels with respect to the conduction-band edge $\left(\Delta E_{\mathrm{HSE06}+\mathrm{V}_{\mathrm{W}}}\right)$ of substitutional and ASV defects of V and W.

\begin{tabular}{lcc}
\hline \hline Defect & $w[\mathrm{eV}]$ & $\Delta E_{\mathrm{HSE06}+\mathrm{V}_{\mathrm{w}}[\mathrm{eV}]}$ \\
\hline $\mathrm{V}_{\mathrm{Si}}:(+\mid 0)$ & -2.7 & -1.69 \\
$\mathrm{~V}_{\mathrm{Si}}:(0 \mid-)$ & -2.2 & -0.94 \\
$\mathrm{~V}-\mathrm{ASV}:(+\mid 0)$ & -4.6 & -1.47 \\
$\mathrm{~V}-\mathrm{ASV}:(0 \mid-)$ & -4.2 & -1.29 \\
$\mathrm{~W}_{\mathrm{Si}}:(+\mid 0)$ & -1.2 & -1.43 \\
$\mathrm{~W}_{\mathrm{Si}}:(0 \mid-)$ & 0.0 & -0.12 \\
$\mathrm{~W}-\mathrm{ASV}:(+\mid 0)$ & -2.6 & -2.44 \\
W-ASV: $(0 \mid-)$ & -2.4 & -0.64 \\
\hline \hline
\end{tabular}

TABLE IV. Chosen common screening parameters $w_{\mathrm{c}}$ for the pair of substitutional and ASV defects of $\mathrm{V}$ and $\mathrm{W}$. The relative stability $\left(E_{\text {rel }}\right)$ of neutral defects is shown as obtained by the HSE06 and HSE06 $+V_{\mathrm{w}}$ functionals.

\begin{tabular}{llcc}
\hline \hline Defect pair & $w_{\mathrm{c}}[\mathrm{eV}]$ & $E_{\mathrm{re}, \mathrm{HSE06}}[\mathrm{eV}]$ & $E_{\mathrm{re}, \mathrm{HSE06}+\mathrm{Vw}}[\mathrm{eV}]$ \\
\hline V-ASV - $\mathrm{V}_{\mathrm{Si}}$ & -3.65 & 1.82 & 1.87 \\
W-ASV - W & -1.9 & 0.03 & -0.15 \\
\hline \hline
\end{tabular}

The obtained screening parameters and the corrected charge transition levels for the considered defects are summarized in Table III, while the chosen common parameters $w_{\mathrm{c}}$ and the corresponding relative stability for the Si-rich condition can be found in Table IV. With these results in hand, it is possible to draw the transitional-level diagram which shows the relative stability of the defects with respect to the Fermi level (Fig. 3). We note that the HSE06 $+\mathrm{V}_{\mathrm{w}}$ functional with the use of common $w_{\mathrm{c}}$ parameter changes the relative stability on the order of $0.1 \mathrm{eV}$, which is negligible in usual cases where the relative stability is on the order of $1 \mathrm{eV}$. By taking into account the position of the charge transition levels we can state that our correction technique produces superior results for transitional-level diagrams of different defects in comparison with conventional calculations using the HSE06 functional.

\section{Partial density of states: correction scheme}

For a deeper understanding of the effect of our correction scheme on the defect orbitals, we plotted the partial density of states of neutrally charged $\mathrm{V}_{\mathrm{Si}}$ in $4 \mathrm{H}-\mathrm{SiC}$ in Fig. 4. Calculations were carried out using both the HSE06 and $\mathrm{HSE06}+\mathrm{V}_{\mathrm{w}}$ functionals. In the case of vanadium $\left(\mathrm{V}^{4+}\right)$ there is only one electron to occupy the lowest double-degenerate $d$-like state $(e)$ that splits into two nondegenerate levels in $\mathrm{C}_{1 \mathrm{~h}}$ symmetry. The major spin channel is chosen to be the

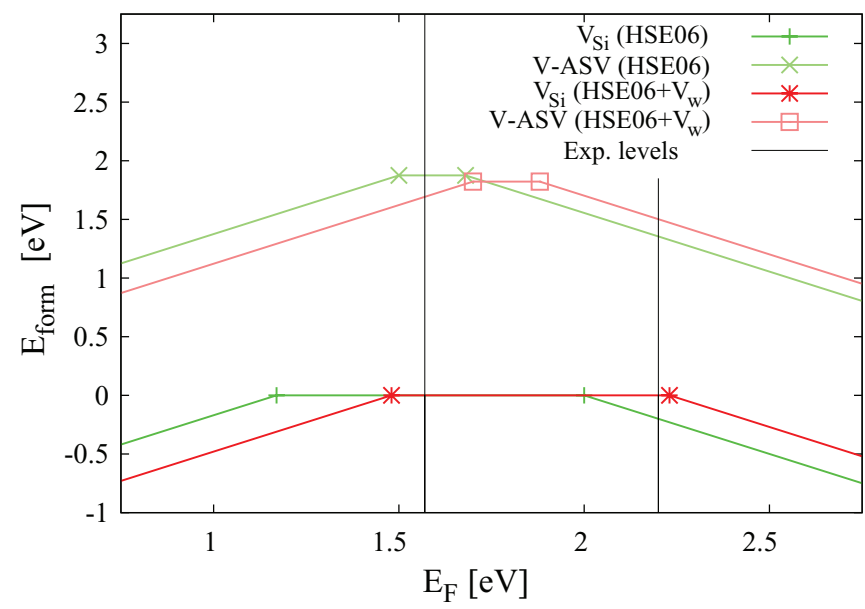

FIG. 3. (Color online) Charge-transition-level diagram of two different types of defects of vanadium (V) in Si-rich conditions, as obtained by the HSE06 and HSE06 $+\mathrm{V}_{\mathrm{w}}$ functionals (see text). Black vertical lines represent the position of the experimental charge transition levels. The formation energy of the substitutional defect was set to zero as a reference energy. 
(a)

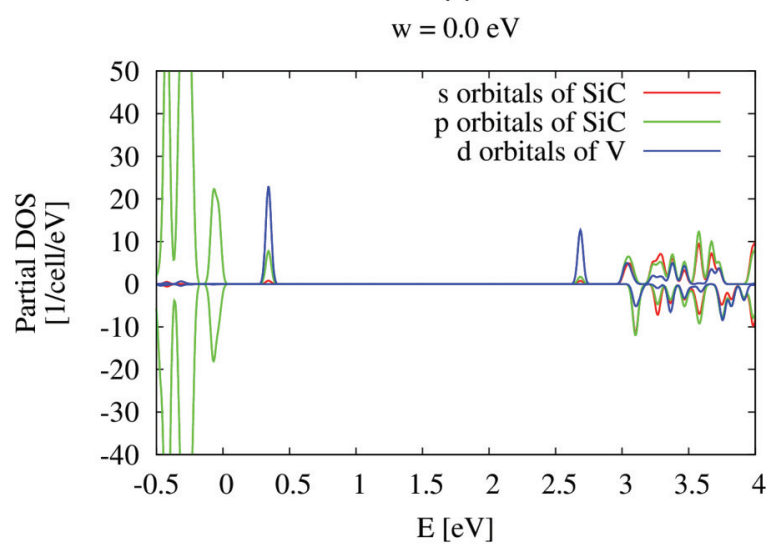

(b)

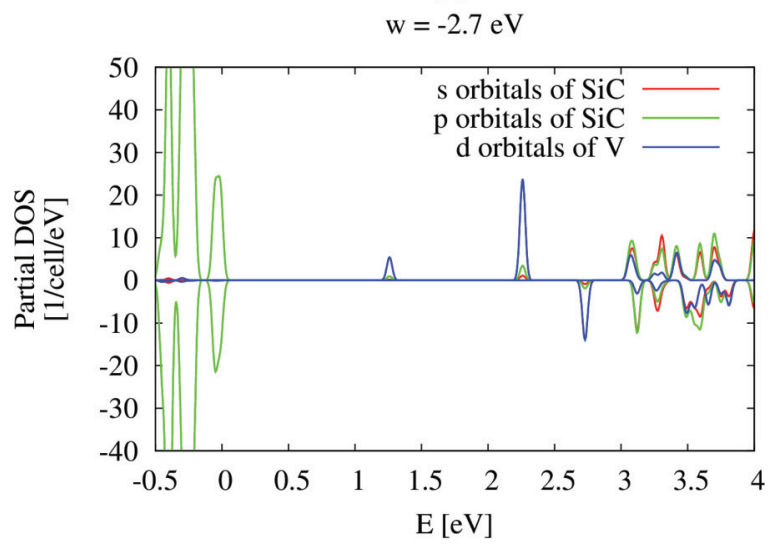

(c)

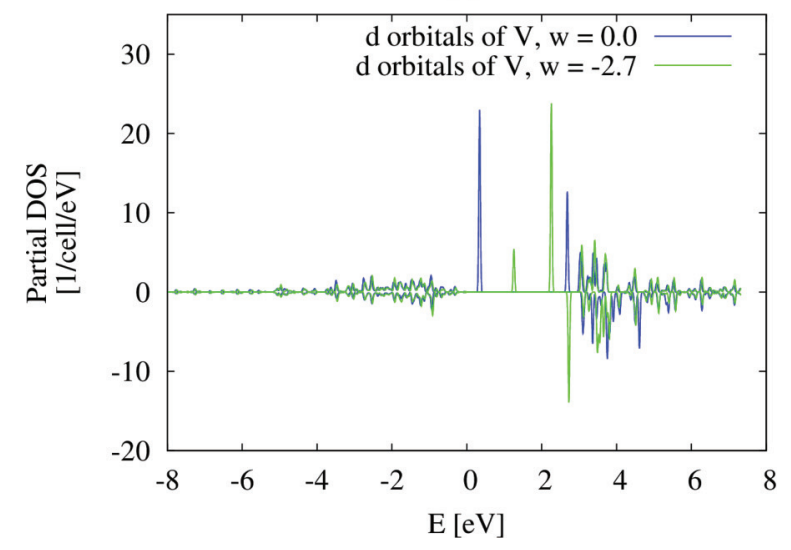

FIG. 4. (Color online) Partial density of states (PDOS) of substitutional vanadium in $4 \mathrm{H}$-SiC calculated by (a) HSE06 functional and (b) and HSE06 $+\mathrm{V}_{\mathrm{w}}$ functional close to the Fermi level; (c) by HSE06 and HSE06 $+V_{w}$ functional in a wide energy range. The energy of the valence band edge was set to zero as a reference energy. Up (down) labels the spin-up (spin-down) channel. The spin-up channel is the majority-spin channel. The calculated Fermi level is at 0.37 and $1.27 \mathrm{eV}$ in HSE06 and HSE06 $+\mathrm{V}_{\mathrm{w}}$ calculations, respectively.

spin-up channel. In the major spin channel both states appear in the gap; the lowest is occupied and the other is unoccupied beause the Fermi-level is at 0.37 and $1.27 \mathrm{eV}$ in the HSE06 and HSE06 $+\mathrm{V}_{\mathrm{w}}$ calculations, respectively. These states are slightly hybridized with the $s$ and $p$ orbitals of the host
TABLE V. Projection of defect states of TM impurities on $d$ orbitals. In all cases the ground state was determined by the HSE06 functional. The integration was done for an impurity-centered sphere of radius $r_{\mathrm{Ti}}=1.32 \AA, r_{\mathrm{V}}=1.22 \AA, r_{\mathrm{W}}=1.46 \AA, r_{\mathrm{Cr}}=1.32 \AA$, and $r_{\mathrm{Fe}}=1.16 \AA$.

\begin{tabular}{lccl}
\hline \hline $\begin{array}{l}\text { Impurity, } \\
\text { charge state }\end{array}$ & Defect state & $\begin{array}{c}\text { Projection on } \\
d \text { orbitals }\end{array}$ & Correction \\
\hline $\mathrm{Ti}^{-}$ & Split $e$ & 0.07 & Not needed \\
$\mathrm{V}^{-}$ & $e$ & 0.60 & Needed \\
$\mathrm{W}^{0}$ & $e$ & 0.50 & Needed \\
$\mathrm{W}^{-}$ & $a$ & 0.08 & Not needed \\
$\mathrm{Cr}^{2-}$ & $e$ & 0.68 & Needed \\
$\mathrm{Cr}^{-}$ & $a$ & 0.33 & Not worked \\
$\mathrm{Fe}^{0}$ & Split $t_{2}$ & 0.32 & Not worked \\
\hline \hline
\end{tabular}

semiconductor. The other states, however, are so strongly hybridized with the $s$ and $p$ orbitals that they lose their atomic character. This is particularly apparent for the occupied states below the Fermi level [see Fig. 4(c)] while empty resonant $d$ states may occur in the conduction band. The effect of the correction potential on those states is almost negligible but very strong on the states occurring in the band gap, as can be seen from Fig. 4. Our proposed correction scheme causes a significant effect on the atomic-like states in the gap; however, it has little effect on the other states. This shows the limitation of this correction technique, which we discuss further in the next section.

\section{E. Applicability of correction scheme}

As we mentioned above our correction technique works properly on truly $d$-like defect states. For an evaluation of the correction it is important to know how the potential acts on the defect state. In this section we investigate this issue and we show that evaluation of two quantities helps to determine the applicability of our correction scheme. The main quantity is the projection of defect states on $d$ orbitals (see Table V). However, this value depends not just on the contribution of the atomic $d$-orbital but also on the localization of the defect state on the TM impurity. In order to separate these two effects we determine the radial distribution of the charge density of the defect state around the TM impurities.

As can be seen in Fig. 5 the degree of localization of the defect states can be separated into two classes. From these two distinct data sets we conclude the following:

(i) If the projection on the $d$ orbital and the localization on the impurity are both small then no correction is needed. This is the case for Ti and the $a$ state of W.

(ii) If the localization is low but the orbitals are quite atomic like (for example $4 d, 5 d$ orbitals) then small correction is needed. That happens to the $e$ state of $\mathrm{W}$.

(iii) If the state is well localized and shows $d$ character then correction is needed; for example, the $\mathrm{V}$ and $e$ states of $\mathrm{Cr}$.

(iv) If the defect state is localized but the contribution of the $d$ orbital to the defect state is low then our correction potential has only partial success and changes the hybridization of the defect state. 


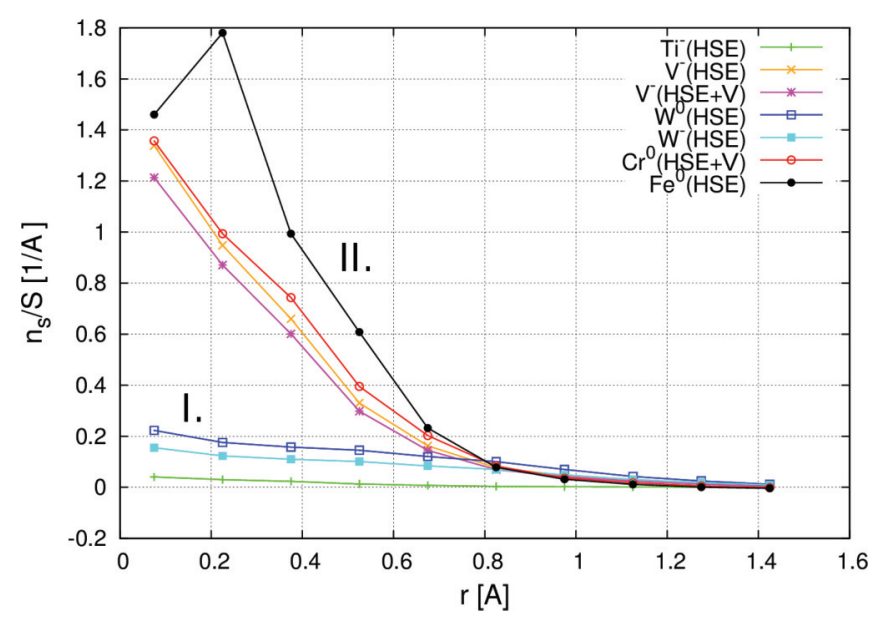

FIG. 5. (Color online) Radial dependence of charge density of defect state around TM defect upon using various functionals. There are two classes of TM impurities. Members of the first class (I) have delocalized defect states while the defect state is much more localized around the TM impurities in the second class (II). Note for the $\mathrm{V}^{-}$defect that the $\mathrm{V}_{\mathrm{w}}$ correction with a negative value will reduce the localization of the defect state, as expected. The defect state of $\mathrm{Cr}^{0}$ appears only with the corrected functional, as explained in the previous section.

\section{F. Identification of $\mathrm{W}$-related defect in $4 \mathrm{H}-\mathrm{SiC}$ by means of corrected functional}

Finally, we show the predictive power of our methodology demonstrated on W-related defects in $4 H$-SiC. We find that the $\mathrm{W}_{\mathrm{Si}}$ defect has two charge transition levels and these are very close to the experimental values. In the case of the $\mathrm{W}_{\mathrm{ASV}}$ defect, HSE06 again suffers from the self-interaction error, $E_{\mathrm{NK}}=-0.18 \mathrm{eV}$, and correction is needed in the functional with $w=-2.4 \mathrm{eV}$. It is worth noticing that this value differs from the value needed for the $\mathrm{W}_{\mathrm{Si}}$ defect, which shows that the self-interaction error for TM defects may be different in various defect configurations. With our correction method the $(0 \mid-)$ charge transition level of the $\mathrm{W}_{\mathrm{ASV}}$ defect is at $\varepsilon_{\mathrm{CBM}}-0.64 \mathrm{eV}$ where no W-related charge transition level was found in experiments (see Table I). This indicates that $\mathrm{W}_{\mathrm{Si}}$ is associated with the measured W-related center, ${ }^{27}$ thus we show the calculated ionization energies of $\mathrm{W}_{\mathrm{Si}}$ in Table I.

\section{CONCLUSION}

In summary, our theoretical investigation reveals that the HSE06 hybrid functional may overlocalize the defect states that arise from transition-metal defects. The reason for this error is the self-interaction error that is increased due to insufficient screening of the Coulomb interaction between electrons localized at the transition-metal defects. By invoking the generalized Koopmans' condition, we are able to indicate the presence of the self-interaction error in the calculation. Furthermore, we suggest a correction method, the HSE06 $+\mathrm{V}_{\mathrm{w}}$ scheme, which can eliminate the self-interaction error of hybrid functionals in the case of atomic-like defect states. With this technique all the calculated charge transition levels become accurate within $0.1 \mathrm{eV}$. In addition, the corrected Kohn-Sham levels are close to those obtained by the sophisticated but computationally demanding $G W_{0}$ method. We demonstrate the applicability of our method for the broad range of problems relevant to the physics of defects in semiconductors.

\section{ACKNOWLEDGMENTS}

Discussions with Rickard Armiento are highly appreciated. Support is acknowledged from the Swedish Foundation for Strategic Research, the Swedish Research Council, the Swedish Energy Agency, the Swedish National Infrastructure for Computing Grants No. SNIC 011/04-8 and No. SNIC00110-223, Knut \& Alice Wallenberg Foundation, EU FP7 project DIAMANT, and the European Science Foundation for Advanced Concepts in ab-initio Simulations of Materials.

\footnotetext{
*vikiv@ifm.liu.se

†agali@eik.bme.hu

${ }^{1}$ C. G. V. de Walle and J. Neugebauer, J. Appl. Phys. 95, 3851 (2004).

${ }^{2}$ D. M. Ceperley and B. J. Alder, Phys. Rev. Lett. 45, 566 (1980).

${ }^{3}$ J. P. Perdew and A. Zunger, Phys. Rev. B 23, 5048 (1981).

${ }^{4}$ Y. Wang and J. P. Perdew, Phys. Rev. B 44, 13298 (1991).

${ }^{5}$ J. P. Perdew, K. Burke, and M. Ernzerhof, Phys. Rev. Lett. 77, 3865 (1996).

${ }^{6}$ P. Deák, B. Aradi, T. Frauenheim, E. Janzén, and A. Gali, Phys. Rev. B 81, 153203 (2010).

${ }^{7}$ S. Lany and A. Zunger, Phys. Rev. B 80, 085202 (2009).

${ }^{8}$ P. Rinke, A. Janotti, M. Scheffler, and C. G. Van de Walle, Phys. Rev. Lett. 102, 026402 (2009).

${ }^{9}$ A. Gali, P. Deák, N. T. Son, and E. Janzén, Appl. Phys. Lett. 83, 1385 (2003).

${ }^{10}$ J. M. Knaup, P. Deák, T. Frauenheim, A. Gali, Z. Hajnal, and W. J. Choyke, Phys. Rev. B 71, 235321 (2005).

${ }^{11}$ A. Gali, P. Deák, P. Ordejón, N. T. Son, E. Janzén, and W. J. Choyke, Phys. Rev. B 68, 125201 (2003).
}

${ }^{12}$ J. M. Knaup, P. Deák, T. Frauenheim, A. Gali, Z. Hajnal, and W. J. Choyke, Phys. Rev. B 72, 115323 (2005).

${ }^{13}$ A. Alkauskas, P. Broqvist, F. Devynck, and A. Pasquarello, Phys. Rev. Lett. 101, 106802 (2008).

${ }^{14}$ J. Paier, M. Marsman, K. Hummer, G. Kresse, I. C. Gerber, and J. G. Ángyán, J. Chem. Phys. 124, 154709 (2006).

${ }^{15}$ V. L. Chevrier, S. P. Ong, R. Armiento, M. K. Y. Chan, and G. Ceder, Phys. Rev. B 82, 075122 (2010).

${ }^{16}$ R. Leitsmann, F. Kuwen, C. Rodl, C. Panse, and F. Bechstedt, J. Chem. Theor. Comput. 6, 353 (2010).

${ }^{17}$ A. Stroppa and G. Kresse, Phys. Rev. B 79, 201201 (2009).

${ }^{18}$ A. Stroppa, G. Kresse, and A. Continenza, Phys. Rev. B 83, 085201 (2011).

${ }^{19}$ S. Lany and A. Zunger, Phys. Rev. B 81, 205209 (2010).

${ }^{20}$ A. Walsh, J. L. F. Da Silva, and S.-H. Wei, Phys. Rev. Lett. 100, 256401 (2008).

${ }^{21}$ I. Dabo, A. Ferretti, N. Poilvert, Y. Li, N. Marzari, and M. Cococcioni, Phys. Rev. B 82, 115121 (2010).

${ }^{22}$ M. Cococcioni and S. de Gironcoli, Phys. Rev. B 71, 035105 (2005). 
${ }^{23}$ M. Schulz, T. Dalibor, W. Martienssen, H. Landolt, and R. Börnstein, Impurities and Defects in Group IV Elements, IV-IV and III-V Compounds, edited by Lamdolt-Börnstein, Vol. 41 (Springer, Berlin, 2003), Subvol. a,2.

${ }^{24}$ Recent Major Advances in SiC, edited by W. J. Choyke, H. Matsunami, and G. Pensl (Springer-Verlag, Berlin, Heidelberg, 2004).

${ }^{25}$ A. Gällström, B. Magnusson, and E. Janzén, Mater. Sci. Forum 615-617, 405 (2009).

${ }^{26}$ M. S. Miao and W. R. L. Lambrecht, Phys. Rev. B 74, 235218 (2006).

${ }^{27}$ F. C. Beyer, C. G. Hemmingsson, A. Gällström, S. Leone, H. Pedersen, A. Henry, and E. Janzén, Appl. Phys. Lett. 98, 152104 (2011).

${ }^{28}$ V. Ivády, A. Gällström, N. T. Son, E. Janzén, and A. Gali, Phys. Rev. Lett. 107, 195501 (2011).

${ }^{29}$ G. Kresse and J. Furthmüller, Phys. Rev. B 54, 11169 (1996).

${ }^{30}$ P. E. Blöchl, Phys. Rev. B 50, 17953 (1994).

${ }^{31}$ C. Freysoldt, J. Neugebauer, and C. G. Van de Walle, Phys. Rev. Lett. 102, 016402 (2009).

${ }^{32}$ L. Hedin, Phys. Rev. 139, A796 (1965).
${ }^{33}$ H. J. Monkhorst and J. K. Pack, Phys. Rev. B 13, 5188 (1976).

${ }^{34}$ S. L. Dudarev, G. A. Botton, S. Y. Savrasov, C. J. Humphreys, and A. P. Sutton, Phys. Rev. B 57, 1505 (1998).

${ }^{35}$ M. A. L. Marques, J. Vidal, M. J. T. Oliveira, L. Reining, and S. Botti, Phys. Rev. B 83, 035119 (2011).

${ }^{36}$ D. O. Scanlon and G. W. Watson, Phys. Chem. Chem. Phys. 13, 9667 (2011).

${ }^{37}$ F. Iori, M. Gatti, and A. Rubio, Phys. Rev. B 85, 115129 (2012).

${ }^{38}$ V. I. Anisimov, J. Zaanen, and O. K. Andersen, Phys. Rev. B 44, 943 (1991).

${ }^{39}$ A. I. Liechtenstein, V. I. Anisimov, and J. Zaanen, Phys. Rev. B 52, R5467 (1995).

${ }^{40}$ P. Novák, J. Kuneš, L. Chaput, and W. E. Pickett, Phys. Status Solidi B 243, 563 (2006).

${ }^{41}$ D. A. Andersson, S. I. Simak, B. Johansson, I. A. Abrikosov, and N. V. Skorodumova, Phys. Rev. B 75, 035109 (2007).

${ }^{42}$ B. Alling, T. Marten, and I. A. Abrikosov, Phys. Rev. B 82, 184430 (2010).

${ }^{43}$ F. Aryasetiawan, M. Imada, A. Georges, G. Kotliar, S. Biermann, and A. I. Lichtenstein, Phys. Rev. B 70, 195104 (2004).

${ }^{44}$ T. Miyake and F. Aryasetiawan, Phys. Rev. B 77, 085122 (2008). 